\title{
FUNDAMENTOS PARA O DESENHO DE ESTRATÉGIAS DE PREVENÇÃO PARA A PROMOÇÃO DA SATISFAÇÃO E DA RESILIÊNCIA CONJUGAL
}

\author{
Ana Maria Vedes ${ }^{1}$ \\ Wolfgang Lind ${ }^{2}$ \\ Madalena Lourenço ${ }^{3}$
}

Resumo: Existe actualmente uma vasta investigação sobre relações amorosas que valida intervenções eficazes com casais. No entanto, em Portugal, o campo da prevenção conjugal carece de fundamentação empírica. Tendo isto em consideração, este artigo pretende: 1) apresentar uma revisão crítica da literatura especializada sobre os principais contributos relativos à educação e enriquecimento conjugal e 2) reflectir sobre aspectos relevantes, ao nível do conteúdo e do processo, para o desenho de estratégias de prevenção para a promoção da satisfação e da resiliência conjugal, em geral, e em particular considerando o contexto português. Foram identificados vários conteúdos pertinentes: a promoção da intimidade, a gestão construtiva de conflitos, o coping diádico positivo, e a consciencialização de diferenças de género relativas a estas dimensões, atendendo a diferentes tipologias de casais felizes. Ao nível do processo, propomos uma cuidada avaliação quantitativa e qualitativa dos perfis de resiliência e de risco dos casais, e a discussão sobre os objectivos a atingir. Consideramos igualmente, a partir da revisão de literatura efectuada, que uma abordagem integrativa - baseada na aplicação de inventários, no feedback, na transmissão de informação e no desenvolvimento de competências relacionais - poderá ser a mais útil, eficaz e eficiente.

Palavras-Chave: Prevenção, Enriquecimento, Satisfação e Resiliência Conjugal.

Grounds for the Design of Prevention Strategies for the Promotion of Couples Satisfaction and Resilience (Abstract): There is currently a vast research in romantic relationships that validates effective interventions with couples. However, in

\footnotetext{
${ }^{1}$ Doutoramento Inter-Universitário em Psicologia Clínica, Psicologia da Família e Intervenções Familiares - Faculdade de Psicologia da Universidade de Lisboa e Faculdade de Psicologia e Ciências da Educação da Universidade de Coimbra. Email: anavedes@gmail.com

2 Faculdade de Psicologia da Universidade de Lisboa

${ }^{3}$ Faculdade de Psicologia e Ciências da Educação da Universidade de Coimbra.
} 
Portugal, the field of couples' prevention lacks empirical validation. Considering this, this article aims: 1) to make a critical review of the literature regarding the main contributions on couples 'education and enrichment, and 2) to reflect on relevant aspects,in terms of content and process, for the development of strategies of prevention and promotion of couples' satisfaction and resilience, in general, and particularly considering the Portuguese context. We considered several contents as relevant: promotion of intimacy, constructive management of conflicts, positive dyadic coping, awareness of gender differences related to the latter dimensions, considering different typologies of happy couples. Regarding the process, we propose a careful quantitative and qualitative assessment of couples resilience and risk profiles, and a discussion on the achievement goals. We also consider, based on this literature review, that an integrative approach - based on the application of inventories, feedback, transmission of information, and development of relational skills - may be the most useful, effective and efficient one.

Key Words: Prevention, Enrichment, Couples`Satisfaction and Resilience

\section{Introdução}

Será que podemos promover e enriquecer a nossa satisfação e resiliência conjugal? Este artigo pretende dar algumas respostas a esta questão tão pertinente para os casais.

Existe actualmente uma vasta investigação sobre relações amorosas que possibilita e valida uma prevenção eficaz com casais (Blanchard, Hawkins Baldwin, \& Fawcett, 2009; Halford \& Moore, 2002; van Widenfelt, Markman, Guerney, Behrens, \& Hosman, 1997). Neste artigo, a partir de uma análise crítica sobre os principais contributos teóricos, interventivos e empíricos no campo da educação e enriquecimento conjugal, pretende-se reflectir sobre os ingredientes que poderão ser mais pertinentes para o desenho de estratégias de prevenção para a promoção da satisfação e da resiliência conjugal, para casais portugueses (casados ou em união de facto).

Assim, após a operacionalização de alguns conceitos-chave, iremos desenvolver os nossos objectivos, começando por reflectir sobre o significado, a importância e a complexidade das relações amorosas e, consequentemente, sobre a importância da prevenção primária e secundária, neste campo; seguidamente faremos uma análise crítica daqueles que consideramos serem os principais contributos teóricos, interventivos e empíricos no campo da educação e enriquecimento conjugal; faremos, ainda, algumas considerações sobre alguns dos principais desafios que a educação e enriquecimento conjugal encara a nível internacional; e, finalmente, fazemos uma proposta de investigação, bem como de conteúdos e processos, que poderão ser fun- 
damentais para o início deste campo de intervenção, empiricamente fundamentado, em Portugal.

\section{Operacionalização de Conceitos Chave}

Apesar dos conceitos de promoção e prevenção poderem ser perspectivados num contínuo, a promoção da saúde definida como: "o processo que habilita os indivíduos e as comunidades a aumentar o seu controlo sobre os determinantes da saúde e, desse modo, melhorarem a sua saúde" (World Health Organization, 1998, p. 2), é um conceito que se foca mais no positivo, no controlo e na melhoria do bem-estar. Na prevenção, o objectivo é diminuir a taxa de incidência ou prevalência de determinadas perturbações (van Widenfelt et al., 1997) ${ }^{4}$. Assim, através da promoção da satisfação e da resiliência conjugal visa-se prevenir possíveis problemas que possam surgir no seio do casal, ou mesmo, a sua ruptura.

Por satisfação conjugal entende-se "uma avaliação subjectiva de dada pessoa em relação ao que sente sobre a sua relação, num dado momento"(Hendrick, Dicke \& Hendrick, 1998, p. 137). A qualidade conjugal diz respeito aos processos conjugais (afectivos, comportamentais e cognitivos) vividos na relação, podendo ser avaliada externamente por um observador com base em critérios que relacionem a qualidade conjugal com a satisfação e/ou sucesso conjugal. Por sucesso conjugal, entende-se a articulação entre qualidade, satisfação e estabilidade conjugal (Narciso, 2001).

A resiliência, enquanto processo de adaptação positiva perante um contexto de adversidade, pode ser entendida como um conceito sistémico, transaccional e dialéctico que compreende as forças do subsistema conjugal - uma vez que: a) se foca mais nos processos através dos quais os indivíduos são mais ou menos resilientes; b) pressupõe que os processos sejam dinâmicos ao longo do tempo; c) reconhece a multiplicidade de acontecimentos ou contextos que têm influências adversas nos sistemas, e d) assegura a diversidade nos resultados obtidos pelos sistemas (ver Conger \& Conger, 2002).

Achamos que o dinamismo inerente ao significado da resiliência capta bem a nossa perspectiva de que é importante intervir preventivamente semeando mudanças de primeira ordem (i.e.,mudanças de natureza quantitativa que ocorrem no interior do sistema sem que os mesmos se alterem), mas

\footnotetext{
${ }^{4}$ Podemos distinguir três tipos de prevenção. A prevenção primária visa reduzir o número de novos casos de problemas conjugais na população em geral; a prevenção secundária visa assistir casais sinalizados/identificados como vulneráveis; e a prevenção terciária (e.g., terapia conjugal) visa tratar e reabilitar casais já com problemas (Bradbury \& Fincham, 1990).
} 
também fomentando forças para mudanças de segunda ordem (i.e., mudanças qualitativas que modificam o próprio sistema; ver Watzlawick, Weakland, \& Fish, 1975).

Neste artigo, focamo-nos na prevenção primária e secundária. Utilizaremos, frequentemente, os termos educação e enriquecimento conjugal como sinónimo de promoção da satisfação e da resiliência conjugal, por serem termos largamente utilizados na literatura.

\section{Significado, importância e complexidade das relações amorosas}

As relações interpessoais íntimas e satisfatórias são, para a maioria dos indivíduos, a fonte de felicidade mais importante, ao constituírem nichos desenvolvimentistas e sociais significativos (Silliman, Stanley, Coffin, Markman, \& Jordan, 2001) e privilegiados para satisfazer as necessidades individuais de afecto, companheirismo, lealdade e intimidade (Halford, Kelly, \& Markman, 1997).

Contudo, o bem-estar que o casamento proporciona está dependente do nível de satisfação conjugal. Quando as pessoas estão insatisfeitas com a sua relação, segundo Ross (1995), os níveis de stress são mais elevados do que nos indivíduos não casados, e mesmo comparativamente com divorciados. Este elevado nível de stress e o mal-estar que as conjugalidades insatisfeitas provocam poderá estar ligado, por um lado, ao aumento dos divórcios na nossa sociedade; e, por outro, à progressiva consciencialização de que a prevenção terciária não é suficiente para travar o aumento de relações disfuncionais (Bradbury \& Fincham, 1990; Christensen \& Heavey, 1999).

Esta consciencialização tem-se manifestado: 1) na atenção crescente que tem sido dedicada ao estudo da conjugalidade ao longo do ciclo de vida do casal (e.g., Karney \& Bradbury, 1995; Lindahl, Malik, \& Bradbury, 1997); 2) no estudo dos factores de risco e factores protectores associados ao divórcio (e.g., Gottman, 1993; Markman, Floyd, Stanley, \& Storaasli, 1988) e às dinâmicas de casais satisfeitos (Halford et al., 1997; Silliman et al., 2001); 3) no desenho, implementação e disseminação de programas que visam prevenir a disfuncionalidade antes que ela se desencadeie. Existem mesmo alguns governos (E.U.A., Austrália, Japão, Noruega) que têm vindo a promover a educação e o enriquecimento conjugal; e 4) na preocupação com a eficácia destes programas (e.g., Halford, Markman, \& Stanley, 2008; Halford \& Moore, 2002;).

Os problemas relacionais não só estão associados a consequências psicológicas e físicas danosas para os adultos como podem ter também efeitos negativos nos filhos (e.g., Christensen \& Heavey, 1999; Gurman, 2009). Assim, parece-nos pertinente afirmar que o alívio dos problemas relacionais 
traz benefícios quer para o casal, quer para as suas famílias, quer para a sociedade em geral (Bradbury \& Fincham, 1990; Floyd, Markman, Kelly, Blumberg, \& Stanley, 1995; Gurman, 2009; Gurman \& Fraenkel, 2002; Halford et al., 2008).

As conjugalidades são extremamente ricas e complexas, espelhando-se tal multidimensionalidade numa série de aparentes paradoxos que todos os casais têm de gerir: a dimensão autonomia versus dependência (Narciso e Ribeiro, 2009); a dimensão estabilidade versus mudança (Alarcão, 2006) e a dimensão segurança versus novidade (Perel, 2007). A forma como os casais gerem estes desafios em função das suas necessidades, é o que faz com que desenvolvam mais as suas forças ou as suas fragilidades (Alarcão, 2006; Narciso \& Ribeiro, 2009).

Um dos maiores desafios que as conjugalidades enfrentam é a sua capacidade de adaptação às mudanças que vão ocorrendo. Segundo Brennan (2001), a adaptação é um processo (que engloba diferentes mecanismos de coping), dinâmico (que passa por várias fases ao longo do tempo), e psicossocial (requer processos psicológicos e sociais), que ocorre à medida que o indivíduo e aqueles com quem ele se relaciona gerem, aprendem e se adaptam a múltiplas mudanças.

Isto constitui também um dos maiores desafios para os investigadores nomeadamente, averiguar como adaptar/construir intervenções que vão ao encontro das diferentes necessidades inerentes ao ciclo de vida do casal (Halford, 2004). Todavia, não obstante estes desafios, a importância e os benefícios da prevenção são inegáveis, como poderemos constatar em seguida.

\section{Importância da prevenção primária e secundária}

A partir da revisão de literatura que realizámos, agrupámos uma série de factores, consoante diferentes formatos de intervenção, que enfatizam a importância da prevenção. Relativamente às intervenções universais - destinadas a casais satisfeitos que, de um modo geral, não experienciam stress significativo (Blanchard et al., 2009) - estas podem: 1) ajudar os casais a fortalecer e/ou adquirir certas competências que tornem a relação uma fonte mais consistente de benefícios, e consequentemente, mais capaz de lidar com as mudanças desenvolvimentistas (Floyd et al., 1995)5; 2) alcançar um grande número de casais, caso sejam aplicadas em formato grupal e em comuni-

\footnotetext{
${ }^{5}$ Dados sobre o divórcio sugerem que, frequentemente, uma disfunção conjugal pode propagar-se durante os primeiros anos do casamento (Rogge, Cobb, Johnson, Lawrence, \& Bradbury, 2002) e que uma elevada percentagem dos divórcios ocorre nos primeiros cinco anos de casamento (Clarke, 1995).
} 
dades; 3) conduzir a ganhos económicos uma vez que a prevenção da disfunção conjugal levaria à diminuição da necessidade de procura de uma série de serviços médicos, psicológicos e legais (van Widenfelt et al., 1997).

As vantagens das intervenções selectivas - destinadas a casais em grande risco de virem a desenvolver problemas relacionais (Blanchard et al., 2009) - consistem em: 1) aumentar a resiliência dos cônjuges face a potenciais adversidades decorrentes de certos factores de risco associados ao divórcio; 2) prevenir alguns factores que são especialmente difíceis de mudar retroactivamente (e.g., memórias de violência e/ou abuso na relação, efeitos negativos resultantes da incapacidade de lidar com conflitos permanentes) e face aos quais muitas vezes a terapia tem dificuldade em ajudar os casais, dado o possível estado de deterioração da relação (Bradbury \& Fincham, 1990; van Widenfelt et al., 1997;Veroff, Kulka, \& Douvan, 1981); e 3) ajudar os casais em determinadas fases de mudança especialmente sensíveis, como, por exemplo: na transição para a parentalidade; no aparecimento de uma doença crónica; no caso de recasamento, etc. (Blanchard et al., 2009; Halford \& Moore, 2002).

Quanto às vantagens das intervenções indicativas - destinadas a casais com algum nível de stress relacional (Blanchard et al., 2009) - estas podem facilitar o alívio do stress e o sofrimento dos cônjuges e das respectivas famílias per se ou mediar o período de vivência de problemas relacionais para o início de terapia de casal (Bradbury \& Fincham, 1990). Este aspecto é ainda mais relevante quando os dados indicam que: a) os casais tendem a esperar pedir ajuda, em média, seis anos depois de terem detectado dificuldades conjugais sérias (Notarius \& Boungiorno, 1992, cit. por Gottman \& Gottman, 1999); b) muitos casais podem sentir menos resistência na adesão a um contexto psico-educativo do que a um setting terapêutico (Halford \& Simons, 2005); e c) casais em lista de espera pioram drasticamente (e.g., Ryan \& Gottman, s.d).

\section{Contributos teóricos}

Existem diferentes maneiras de perspectivar as relações íntimas adultas (Gurman, 2009; Gurman \& Fraenkel, 2002; Narciso \& Ribeiro, 2009). Contudo, parece haver uma tendência para perspectivar a conjugalidade como processo (Narciso \& Ribeiro, 2009) e para identificar, elucidar e aplicar princípios teórico-práticos comuns nesta área (ver Gurman, 2009). Iremos, assim, abordar mais especificamente os modelos teóricos sobre a conjugalidade que consideramos mais pertinentes, dada a sua riqueza conceptual e empírica e explicitaremos quais as ideias que fomos respigar a estes modelos. 


\section{Teoria da Construção de um Casamento Saudável 6 de Gottman}

Gottman e colaboradores (e.g., Gottman et al., 2002; Gottman \& Gottman, 2009a) desenvolveram um modelo desenvolvimentista, sistémico, integrativo e empiricamente validado sobre o que faz uma relação ser bem sucedida. Segundo o autor, nas relações satisfeitas, os casais conseguem que os comportamentos, pensamentos e sentimentos positivos prevaleçam sobre os negativos - "Dominância do Sentimento Positivo"(Gottman \& Gottman, 2009; p. 140). Assim, casamentos satisfeitos e estáveis ou emocionalmente inteligentes (Gottman \& Silver, 2001) caracterizam-se: 1) pelo afecto positivo - esta é a única variável que prediz concomitantemente a satisfação e a estabilidade conjugal; 2) pela consonância entre os cônjuges da forma como lidam com os conflitos; 3) pelo reconhecimento da inevitabilidade dos conflitos e de que existem problemas que nunca se resolvem (segundo os autores $69 \%$ são problemas permanentes - relacionados com diferenças fundamentais relativas à personalidade ou a necessidades básicas - e apenas $31 \%$ das discussões correspondem a questões resolúveis). No entanto, os casais felizes não ficam presos nas suas posições díspares, procurando encontrar compromissos que satisfaçam ambas as partes através de formas de resolução de conflito funcionais e afectuosas: a) iniciam as discussões de forma suave e positiva; b) deixam-se influenciar; c) são bem sucedidos nas tentativas de reparação; d) conseguem que o processo de diminuição da negatividade funcione; e) evitam usar os "quatro cavaleiros do apocalipse" (i.e. crítica global, atitude defensiva, menosprezo e fuga; Gottman \& Silver, 2001; p. 41) e a beligerância e hostilidade dada a sua perigosidade.

Segundo o modelo proposto por estes autores, o que faz o sucesso de um casamento é uma profunda amizade/intimidade, por ser a melhor arma contra a negatividade em relação ao parceiro e contra a perda de controlo nos conflitos (e.g., Gottman \& Gottman, 2009a). A este modelo iremos buscar aspectos chave da satisfação e do sucesso conjugal relacionados com os processos dos conflitos e da amizade/intimidade.

\section{Modelo Sistémico-Transaccional de Stress e Coping de Bodenmann}

Dos diferentes modelos de coping diádico que existem, escolhemos o Modelo de Bodenmann (2005) pela sua natureza sistémica, integrativa, transaccional e pelo foco nas relações amorosas íntimas. Para Bodenmann (2005) o stress e o coping diádico são partes de um processo interpessoal que envolve ambos os membros do casal e que ocorre num determinado contexto (temporal e espacial). O stress diádico é o encontro com um factor

\footnotetext{
${ }^{6}$ No original The Sound Of Marital House Theory.
} 
stressante específico que afecta ambos os parceiros directa ou indirectamente. O stress afecta a satisfação, a qualidade e o sucesso conjugal de diversas formas: a) pode diminuir o tempo que o casal passa em conjunto e, consequentemente a disponibilidade para estabelecer e concretizar o sentido e sentimento de pertença do casal, b) pode diminuir a qualidade da comunicação diádica - num estudo realizado em laboratório, a qualidade da comunicação relacional sob condições de stress diminuía em $40 \%$-, e c) pode aumentar o risco dos indivíduos desenvolverem problemas físicos e psicológicos (Bodenmann, 2005).

Segundo o autor, 1) não se pode analisar a avaliação de stress ou os esforços de coping de um dos elementos sem considerar os efeitos no outro e no casamento; 2) o coping diádico é apenas uma das várias formas que existem para lidar com o stress; o coping individual e o recurso ao apoio da rede social são outras formas de coping; 3 ) o coping diádico é geralmente usado quando o coping individual já foi accionado e falhou; 4) o coping diádico engloba mecanismos de coping positivo (i.e., apoiante, em conjunto e delegado) e negativo (i.e., hostil, ambivalente e superficial), que podem orientar-se para o problema ou para a emoção.

Tendo em conta o nível de stress que marca a sociedade ocidental actual e a inevitabilidade do stress quotidiano, o coping diádico é de vital interesse para que o casal, em conjunto, consiga encontrar mecanismos de coping para proteger a relação dos efeitos negativos do stress (e.g., o stress crónico diário). Os programas de educação e enriquecimento conjugal devem assim promover estratégias de coping diádico positivo que aumentem a resiliência conjugal (Kayser \& Scott, 2008; Pihet, Bodenmann, Cina, Widmer, \& Shantinath, 2007; Ryan \& Gottman, s/d) e habilitem a díade a lidar melhor com as mudanças.

A este modelo de Bodenmann (2005) iremos buscar as estratégias de coping diádico positivo que consideramos serem de suma importância para uma maior eficiência das intervenções, para uma melhor gestão do paradoxo estabilidade versus mudança, e para promover a resiliência conjugal.

\section{Modelo Sociocognitivo da Comunicação Conjugal de Fitzpatrick}

O Modelo de Fitzpatrick (1988), permite identificar uma tipologia exaustiva e mutuamente exclusiva de casais satisfeitos. Esta teoria tem por base diferentes dimensões conceptuais que são usadas pelos casais para descrever o seu relacionamento: ideologia do tradicionalismo, ideologia da incerteza e da mudança, partilha, regularidade temporal, espaço indiferenciado, autonomia, assertividade e evitamento do conflito. A autora desenvolveu um instrumento para operacionalizar cada uma destas dimensões Relationship Dimension Inventory - agrupando os relacionamentos numa 
tipologia de três definições relacionais distintas: Tradicional, Independente e Separado; que quando conjugando as definições de homens e mulheres originam diferentes tipos conjugais. Parece ser o tipo conjugal (ou seja, a conjugação entre a definição relacional da mulher e a definição relacional do marido) e não a definição individual da relação que regula as interacções conjugais. Consoante o tipo conjugal também varia o critério que constitui um casamento satisfeito.

Estes diferentes esquemas (cognições) conjugais afectam os processos afectivos e comportamentais, que: 1) poderão ter implicações importantes para as intervenções preventivas com casais; 2) podem ser extremamente úteis na avaliação inicial do processo de intervenção e posterior desenho e aplicação de estratégias de educação e enriquecimento conjugal (Ribeiro, 2002), e 3) consideramos ser um ponto-chave que tem sido descurado nesta área.

\section{Contributos interventivos}

A educação e enriquecimento conjugal teve a sua origem nas instituições religiosas (Hunt, Hof, \& DeMaria, 1998) e continua em muitos países ocidentais a ser proporcionada em contextos religiosos (Halford, 2004). Nomeadamente, em Portugal, será um dos poucos contextos, onde é realizada.

Os programas de educação e enriquecimento conjugal variam em diversas dimensões, mas todos visam fortalecer a relação, promover e/ou construir capacidades interpessoais e prevenir o stress conjugal (Bowling, Hill, \& Jencius, 2005).

Segundo Halford e Moore (2002), podemos diferenciar a educação e enriquecimento conjugal em três estratégias principais: 1) abordagens centradas na informação e no conhecimento; 2) abordagens centradas na avaliação ou em inventários e 3) abordagens focadas no treino de competências. No entanto, a maioria das intervenções de educação e enriquecimento conjugal usam elementos das três abordagens (Silliman et al., 2001).

As abordagens centradas no conhecimento, enfatizam a transmissão da informação, clarificação de expectativas e a progressiva consciencialização dos processos relacionais chave que influenciam a qualidade e sucesso da relação. Recorrem para tal, sobretudo a demonstrações, não havendo geralmente treino activo de competências. Este tipo de abordagem tem subjacente o princípio da auto e hetero consciencialização. Podemos apontar diversas limitações a estas abordagens. Por exemplo: a) frequentemente o conteúdo e o processo dos programas não estão bem documentados e faltam-lhes bases conceptuais, empíricas e medidas estandardizadas; b) não está também previsto um espaço para adquirir in loco as novas competências; e, c) os conteúdos poderão não ir ao encontro das necessidades específicas dos 
grupos-alvo (Halford \& Moore, 2002). Contudo, estas abordagens poderão ser úteis em intervenções universais.

Nas abordagens centradas em inventários, geralmente, cada elemento do casal preenche um inventário que avalia uma série de dimensões consideradas a priori como fundamentais para o funcionamento conjugal, e que possam servir de base para a consciencialização, conhecimento, e/ou treino de competências. Alguns investigadores/técnicos usam os inventários como uma forma de pré-diagnóstico das necessidades do casal, definindo com o casal os objectivos específicos de aprendizagem para o posterior desenvolvimento de actividades experienciais. Outros técnicos, usam os inventários apenas como base para consciencializar a díade de aspectos relevantes e, posteriormente, fornecem feedback (Halford \& Moore, 2002). Segundo Silliman e colaboradores (2001), no caso de este tipo de abordagem se basear apenas no feedback de informação relativa à compatibilidade (e.g., personalidade, atitudes, percepções) pode conduzir a uma discussão construtiva e enriquecedora, mas também pode ser deletéria para casais com défices de capacidades de resolução de problemas ao aumentar a ansiedade inerente, à percepção de incapacidade para lidar com as diferenças tornadas salientes pela avaliação.

Existem diversos inventários disponíveis: o Premarital Personal and Relationship Evaluation (PREPARE; Olson, Foumier, \& Druckman, 1996); o Facilitating Open Couple Communication Understanding and Study (FOCCUS; Markey \& Micheletto, 1997) e o Preparation for Marriage (PREP-M; Holman, Larson, \& Harmer, 1994), os quais foram considerados por Larson, Holman, Klein, Busby, Stahmann e Peterson (1995) como os mais consistentes psicometricamente. Ainda que estes inventários tenham sido desenhados para intervenções pré-conjugais, a maioria dos autores desenvolveu versões destinadas a casais já formados (e.g., o ENRICH, corresponde ao PREPARE). Dois questionários que consideramos particularmente pertinentes são: o Sound of Relationship House Questionnaires (SRHQ; Gottman, 1999) e o Dyadic Coping Inventory (DCI; de Bodenmann, 2007), pois foram desenvolvidos com base na vasta investigação de Gottman e de Bodenmann, respectivamente.

Esta abordagem tem proliferado em diversos países ocidentais e apresenta algumas vantagens. Assim, para o FOCCUS, PREPARE e RELATE diversos estudos apontam para que os três inventários predizem a satisfação relacional nos primeiros anos de casamento; logo, medem factores relevantes para o sucesso relacional (Halford, 2004). Uma outra vantagem, é que estes inventários (construídos com base na vasta literatura existente sobre factores determinantes da satisfação e estabilidade conjugal) possibilitam, quer ao investigador quer ao casal, aceder aos perfis de resiliência e risco da díade. Existe também um treino estruturado sobre a aplicação destes instrumentos. 
A principal desvantagem desta abordagem é a inexistência de avaliação sistemática sobre os efeitos da administração dos inventários e do feedback. Abordámos já anteriormente este aspecto dos potenciais efeitos nefastos que um mero feedback pode ter no casal. Uma segunda desvantagem prende-se com a exclusiva análise do casal com base em medidas de auto-relato, o que pode não permitir o acesso a dimensões importantes, não contempladas pelos inventários, que predizem o insucesso conjugal (Halford, 2004).

Face às abordagens focadas no treino de competências, o seu postulado principal é que algumas pessoas não possuem no seu repertório (ou esqueceram) certas competências cruciais na promoção de relações íntimas satisfeitas e de qualidade, porque foram, por exemplo, expostas na família de origem a modelos ineficazes (Halford \& Moore, 2002).

Estas abordagens enfatizam, assim, o ensino dessas competências. Existem múltiplos programas de educação e enriquecimento conjugal dos quais destacamos a) o Relationship Enhancement (RE; Guerney, 1987), b) o Prevention and Relationship Enhancement Program (PREP; Markman et al., 1988), c) o Couples Communication Program (CCP; Miller, Miller, Nunnally, \& Wackman,1992), d) o Couple Commitment Relationship Enhancement Program (Couple CARE; Halford, Moore, Wilson, Dyer, \& Farrugia, 2004, e) o The Art \& Science of Love: a Workshop for Couples (ASL; Gottman \& Gottman, 2006a), e f) o Couples Coping Enhancement Training (CCET; Bodenmann \& Shantinath, 2004) ${ }^{7}$.

Estes programas envolvem uma série de elementos: treino de competências, conferências, demonstrações, apresentações audiovisuais, ensaio e feedback, trabalhos de casa, etc. Relativamente aos conteúdos, as semelhanças são maiores do que as diferenças. RE, PREP, CCP, Couple CARE e CCET trabalham a comunicação positiva, a gestão de conflitos e a expressão positiva de afecto. No entanto, o RE enfatiza sobretudo o desenvolvimento da empatia; o PREP a prevenção dos conflitos negativos; o CCET, o coping individual e diádico com o stress; o Couple CARE, o papel da auto-regulação; e o ASL, a amizade/intimidade, o significado partilhado e formas construtivas de lidar com os conflitos.

\footnotetext{
${ }^{7}$ Segundo Jakubowski, Milne, Brunner e Miller (2004) tendo em conta os critérios de eficácia definidos pela American Psychological Association (i.e., a eficácia ter sido demonstrada em duas amostras de controlo aleatórias conduzidas por duas equipas diferentes de investigadores; existir um manual de tratamento e as publicações serem no formato revisto por pares) o RE é o programa mais eficaz. No entanto, o PREP é o único com dados que provam melhorias no funcionamento conjugal seis meses depois do pós-teste. O CCP, o RE e o PREP foram considerados eficazes. e o Couple CARE e o CCET foram considerados como potencialmente eficazes (Jakubowski, Milne, Brunner, \& Miller, 2004). Sobre a eficácia do ASL, existe apenas um artigo on-lineno site do Gottman Institute (ver http://www.gottman.com/research/projects/couples/).
} 
A maior vantagem desta abordagem é que o treino de competências incide sobre variáveis associadas ao sucesso relacional. No entanto, existe um debate aceso sobre alguns aspectos. Vários estudos indicam que a utilização ou não utilização de determinadas capacidades (e.g., activação elevada de afectos negativos e formas construtivas comunicacionais) no contexto conjugal, pode ser condicionada por múltiplos factores (e.g., atribuições disposicionais negativas, desinvestimento emocional, más recordações, falta de compromisso). Por exemplo, Vincent, Weiss e Birchler (1975) verificaram que determinadas pessoas em relações insatisfeitas e com fracas capacidades comunicacionais não demonstram as mesmas incapacidades noutros contextos.

Por outro lado, não existe consenso empírico sobre quais os aspectos específicos comunicacionais que predizem a insatisfação e o stress conjugal e mesmo se o ensino de competências comunicacionais contribuirá para uma maior satisfação conjugal consistente ao longo do tempo (Spitsberg, 2003). Há, ainda, autores que defendem que o treino de competências sem outro tipo de conteúdos associados (e.g., promoção da intimidade, do compromisso, do afecto, da empatia, da amizade, da gestão de questões relacionadas com a família de origem) é, por si só, insuficiente para promover relações conjugais positivas (Halford \& Moore, 2002; Fincham, Stanley, \& Beach, 2007).

Outras vantagens das abordagens focadas no treino de competências são: 1) a existência substancial de estudos que comprovam a sua eficácia na mudança das competências relacionais pelo menos a curto prazo e 2) o treino estandardizado dos programas que tem conduzido à sua disseminação com eficácia. Todavia, a evidência destas abordagens continua limitada pelos poucos estudos que existem sobre a sua eficácia a longo prazo (i.e., follow ups para além de 12 meses) e os existentes limitam-se ao PREP ou suas variantes (Halford, 2004).

Estas diferenças e semelhanças entre os programas levantam a questão sobre o que é que se sabe sobre os determinantes da satisfação e estabilidade conjugais.

\section{Contributos empíricos}

Existem mais de 200 estudos longitudinais publicados que tentam predizer a satisfação, a qualidade, a estabilidade e o sucesso conjugal (ver para uma revisão da literatura: Halfold, 2004; Halford \& Moore, 2002; Halford, Markman, Kline, \& Stanley, 2003; Karney \& Bradbury, 1995; Lindahl et al.,1997; Markman et al.,1988). Estes estudos, segundo Halford e Moore (2002), sugerem quatro grandes classes de variáveis com impacto no desenvolvimento da satisfação conjugal ao longo do tempo: 1) processos adaptativos do casal (i.e., processos afectivos, comportamentais e cognitivos que 
ocorrem durante as interacções conjugais); 2) transições ao longo do ciclo de vida (i.e., mudanças desenvolvimentistas normativas e não normativas que afectam o casal ou um dos seus elementos); 3) características individuais (i.e., factores estáveis relativos à história, à personalidade e experiências que os indivíduos trazem para a relação) e 4) variáveis contextuais (i.e., família de origem, rede social e trabalho; história da relação e factores culturais).

Estas variáveis podem ser conceptualizadas segundo duas categorias: a) indicadores de risco estático e b) indicadores de risco dinâmico. Os primeiros são factores de risco que não podem ser mudados com uma intervenção, particularmente: 1) idade; 2) experiências na família de origem: o divórcio parental na família de origem, sobretudo nas mulheres; o testemunho de violência entre aos pais, sobretudo nos homens, aumenta o risco de eles próprios virem a ser violentos; 3 ) número de casamentos anteriores; 4) a existência de filhos; 5) a aprovação da união pela família alargada e pelos amigos do parceiro; 6) a relação com o trabalho; 7) o tempo de namoro; 8) a coabitação antes do casamento; 9) a baixa religiosidade; 10) historial de doenças psicológicas; e 11) o contexto cultural e a origem cultural dos membros do casal ${ }^{8}$ (Halford \& Moore, 2002).

Os indicadores de risco estático apresentam a vantagem de poderem ser medidos e despistados com facilidade. A avaliação destes factores pode ajudar os investigadores/interventores a avaliar o nível de risco de um determinado casal e, assim, ajudar a delinear intervenções para quem mais precise - intervenções selectivas (Halford \& Moore, 2002).

Os indicadores de risco dinâmicos são indicadores com maior potencial de mudança, mas mais difíceis de avaliar. Nomeadamente: 1) défices na comunicação e em comportamentos de gestão dos conflitos; 2) expectativas e crenças irrealistas - sobre a importância da comunicação, métodos de resolução dos conflitos, a importância da família, dos amigos e papéis de género; 3 ) atribuições negativas disposicionais; 4) formas destrutivas de resolução de conflitos; 5) pouca intimidade; 6) padrões de expressão emocional negativa: crítica global, desprezo, repugnância, postura defensiva, fuga emocional; e 7) estratégias de coping ineficazes com o stress. Todavia, alguns indicadores de risco dinâmicos aparecem, frequentemente, associados aos indicadores de risco estático, o que permite uma porta de entrada para a sua avaliação - e.g., a comunicação negativa ou estilos de resolução de conflitos não construtivos aparecem associados ao divórcio parental e à agressão na família de origem (Halford \& Moore, 2002).

Existem diferenças significativas entre homens e mulheres na forma como vivenciam os processos relacionais (e.g., intimidade/proximidade, im-

\footnotetext{
${ }^{8}$ Desenhar programas sensíveis às tradições culturais parece ser relevante, sobretudo numa sociedade cada vez mais multicultural (Lind, 2008).
} 
portância da comunicação, forma de resolução de conflitos, relação com o trabalho, conjugação de papéis familiares, iniciativa quanto ao divórcio, vivência e avaliação da satisfação conjugal, sexualidade, estratégias de coping (e.g., Markman \& Kraft, 1989; Narciso, 2001; Narciso \& Ribeiro, 2009) que devem ser alvo de atenção nas intervenções de Educação e Enriquecimento Conjugal (e.g., Halford \& Moore, 2002).

Geralmente é durante períodos de grande mudança ou altamente stressantes que os problemas relacionais têm mais probabilidade de se desenvolver (Karney \& Bradbury, 1995). Todavia, os dados não são consensuais, havendo casais que vivem estas transições como aumentando a satisfação, o compromisso e a proximidade (Halford, Scott, \& Smythe, 2000), o que leva a crer que outras variáveis terão efeitos indirectos nesta relação entre eventos significativos e problemas relacionais. No entanto, quanto menos indicadores de risco dinâmico e estático os casais possuírem, mais resilientes parecem ser (Halford et al., 2003; Markman, Halford, \& Cordova, 1997). Deste modo, a fase de constituição do casal (casamento ou união de facto) parece ser uma boa altura para intervir tendo em conta os desafios que o casal geralmente enfrenta. Certos momentos chave, associados a um maior risco (e.g., transição para a parentalidade, aparecimento de uma doença grave, desemprego) bem como casais com necessidades especiais (i.e., casais que decidem adoptar um filho, casais em que um dos parceiros tem uma doença psicológica e casamentos reconstituídos) também devem ser alvo de intervenção. Estas parecem ser também as alturas em que os próprios casais estão mais receptivos à Educação e Enriquecimento Conjugal (Halford, 2004; Markman et al., 1997).

No entanto, independentemente da fase do ciclo de vida em que se intervenha, a maioria dos estudos refere a necessidade de sessões posteriores de manutenção (e.g., Markman et al., 1997; Ryan \& Gottman, s.d.) o que nos leva à reflexão sobre a eficácia e eficiência das intervenções. A investigação com base na eficácia remete para resultados obtidos em meios controlados. A investigação com base na eficiência está relacionada com resultados obtidos no contexto natural dos sujeitos. A questão principal prende-se em saber até que ponto os resultados da eficácia podem ser generalizados a contextos de eficiência (Christensen \& Heavey, 1999).

\section{Avaliação e desafios actuais no campo da educação e enriqueci- mento conjugal}

Os efeitos da educação e enriquecimento conjugal começaram a ser investigados na década de setenta (Hunt et al., 1998). Diversas revisões de literatura e meta-análises (ver Bradbury \& Fincham, 1990; Halford, 2004; e Halford et al., 2003) têm revelado resultados que apontam para a eficácia da 
educação e enriquecimento conjugal em conduzir a mudanças nos comportamentos durante algum período de tempo e a produzir aumentos moderados no ajustamento e estabilidade conjugal, quer em intervenções universais, selectivas e indicativas (Blanchard et al., 2009), quer em abordagens centradas na avaliação ou em inventários, quer nas abordagens focadas no treino de competências (Halford, 2004; Halford \& Moore, 2002; Halford et al., 2008).

Contudo, o grau de confiança nestes resultados ainda não é claro por diversas limitações metodológicas e dilemas ao nível da investigação. Por exemplo: ausência de consenso relativamente ao efeito médio das intervenções (ver Bradury \& Fincham, 1990; Christensen \& Heavey, 1999); discrepâncias obtidas, consoante se avaliam competências relacionais ou aspectos relativos à satisfação relacional; limitações relacionadas com os efeitos de medida em medidas observacionais e de auto-relato (ver Blanchard et al., 2009; Christensen \& Heavey, 1999); alguns autores defendem que os critérios subjacentes à avaliação dos resultados da educação e enriquecimento conjugaldevem ser alargados (ver Christensen \& Heavey, 1999; Halford, 2004; Halford et al., 2003; Jakubowski et al., 2004; Markman et al., 1997; Stanley, 2001); a questão da durabilidade dos efeitos (ver Bowling et al., 2005; Christensen \& Heavey, 1999); o pouco que se sabe sobre o efeito de variáveis mediadoras (ver Hawkins, Blanchard, Baldwin \& Fawcett, 2008; Jakubowski et al., 2004; Markman et al., 1997); a necessidade de incorporação de mecanismos de motivação para aumentar a adesão de casais satisfeitos (ver Duncan, Holman \& Yang, 2007; Halford \& Moore, 2002; Silliman et al., 2001); o ainda incipiente conhecimento das expectativas dos casais face aos programas (Halford \& Moore, 2002) e dos factores relevantes para a variabilidade na procura de ajuda (Bradbury \& Fincham, 1990; Duncan et al., 2007).

Adicionalmente, a investigação no campo da educação e enriquecimento conjugal enfrenta actualmente diversos desafios: 1) Como conciliar programas estruturados, com a flexibilidade requerida pelas necessidades de cada casal, diferentes estilos de conjugalidade e diferentes perfis de risco? Como responder a esta questão consoante as intervenções? (Blanchard et al., 2009; Christensen \& Heavey, 1999; Hawkins et al., 2008), 2) Como lidar com a pressão de agir já face aos problemas sociais relativos ao stress relacional e ao divórcio, com a necessidade de se saber mais sobre a própria educação relacional? (Stanley, 2001), e 3). Deverão os governos apoiar a educação e enriquecimento conjugal e que cuidados devem ser tidos em conta na sua disseminação? (ver Halford et al., 2008).

Estas são questões ainda sem resposta consensual no panorama internacional. No entanto, podemos procurar ter em conta estes desafios quando pensamos no desenho de estratégias de educação e enriquecimento conjugal em Portugal. 


\section{Ingredientes pertinentes para a educação e enriquecimento con- jugal}

Em Portugal, onde no campo da educação e enriquecimento conjugal está ainda quase tudo por fazer, parece-nos de suma relevância:1) criar um protocolo forte que permita avaliar indicadores de risco estático e dinâmico,e possibilite uma análise dos perfis de risco/resiliência dos casais - assim, construímos (Vedes, Lind, \& Lourenço, 2009) um instrumento que visa avaliar dados sociodemográficos e indicadores de risco estático para o sucesso conjugal, adaptámos para a população portuguesa o Dyadic Coping Inventory (Bodenmann, 2007) e a versão breve do Sound Relationship Questionnaires (Gottman \& Gottman, 2006b); 2) ouvir as vozes de casais com diferentes ideologias conjugais, através do formato Focus Group, sobre as suas necessidades em temos de conteúdo, processo e formato face a um programa de educação e enriquecimento conjugal; e 3) desenvolver estratégias de intervenção com base no cruzamento dos dados recolhidos através dos passos anteriores.

\section{Considerações relativas ao conteúdo}

Tendo como fundamentação os contributos teóricos, interventivos e empíricos que analisámos, consideramos que o Desenho de Estratégias de Prevenção para a Promoção da Satisfação e Resiliência Conjugal deverá, ao nível do conteúdo, ter como objectivos primários: 1) promover a intimidade, dado o papel fulcral que esta possui na satisfação/qualidade conjugal. Perspectivamos o constructo de intimidade como um conjunto de processos afectivos, cognitivos e comportamentais dinâmicos e entrelaçados (partilha, auto-revelação, apoio, confiança, mutualidade, interdependência, e sexualidade), pelos quais o casal se conhece, apoia, se reconstrói no outro e se torna inter(in)dependente (ver Narciso, 2001, 2002). Esta definição dos componentes da intimidade, segundo Narciso (2002) pode ser associada às competências que Gottman refere que os casais emocionalmente inteligentes possuem (e.g., Gottman, Gottman \& DeClair, 2006); 2) promover competências para a resolução de conflitos solucionáveis e para a gestão ou aceitação de conflitos perpétuos, bem como evitar formas destrutivas de comunicação. Vários estudos demonstram que a forma como os casais lidam com as situações de conflito é o melhor preditor do sucesso da conjugalidade a longo prazo (e.g., Gottman et al., 2006); 3) promover o coping diádico positivo. A ausência de coping ao nível da díade é um dos principais preditores do divórcio (Bodenmann, 1997; Hahlweg, Markman, Thurmaier, Engl, \& Eckert, 1998; Jacobson, Schmaling \& Holtzworth-Munroe, 1987); 4) atender a diferentes tipologias de casais satisfeitos, com diferentes objectivos e dife- 
rentes perfis de risco e resiliência (Halford, 2004) sob pena de se continuar na redundância da classe média branca, de um formato interventivo one size fits all e no apanágio de um protótipo de casamento ideal (ver Hendrick, 2002) e 5) consciencializar para certas dinâmicas conjugais relacionadas com as diferenças de género (e.g., Halford \& Moore, 2002). ${ }^{9}$

\section{Considerações relativas ao processo}

Quanto ao processo, consideramos que uma maior eficácia e eficiência, poderá ser alcançada caso o Desenho de Estratégias de Prevenção para a Promoção da Satisfação e Resiliência Conjugal se baseie: 1) numa cuidada avaliação, que utilize métodos quantitativos (i.e., a partir de diversos inventários que avaliem as dimensões por nós anteriormente mencionadas bem como indicadores de risco estático) e qualitativos (i.e., entrevista sobre o início, história relacional e recursos do casal) (Blanchard et al., 2009; Christensen \& Heavey, 1999; Halford, 2004; Hawkins et al., 2008); 2) numa discussão com o casal sobre os aspectos relacionais que visam abordar/trabalhar, e de que forma (Halford \& Moore, 2002; Hawkins et al., 2008); 3) em estratégias integrativas, que utilizem componentes educativas, feedback e treino de competências (Siliman et al., 2001; Hawkins et al, 2008; Ryan \& Gottman, s.d.); 4) em prescrições que promovam a reprodução e insight do casal no seu contexto natural, bem como numa avaliação ao longo de diversos momentos temporais da satisfação e da resiliência conjugal e, de acordo com esta informação, desenhar e aplicar sessões de reforço (Blanchard et al., 2009; Bowling et al., 2005; Markman et al., 1997; Ryan \& Gottman, s.d.).

\section{Conclusão}

Vimos ao longo do artigo que é possível promover a satisfação e a resiliência conjugal. Consideramos, que as nossas propostas poderão ser as mais eficazes e eficientes para o início da educação e enriquecimento conjugal, desde que empiricamente fundamentadas em Portugal, proporcionando directrizes que procuram responder a diferentes tipos de intervenções, diferentes perfis de risco e resiliência conjugal, diferentes conjugalidades satisfeitas e às expectativas e necessidades dos casais. Gostaríamos de realçar a

\footnotetext{
${ }^{9}$ A comunidade científica sugere alguns conteúdos adicionais, mas com uma importância mais secundária, tais como: promover o compromiso, ou seja, o investimento na continuidade da relação (Clements, Stanley, \& Markman, 2004; Narciso \& Ribeiro, 2009); e promover padrões cognitivos conjugais realistas, dado o papel que expectativas parecem ter na satisfação conjugal e sucesso conjugal (Narciso \& Ribeiro, 2009).
} 
urgência em iniciar a investigação e intervenção neste campo,em Portugal, porque uma maior satisfação conjugal beneficia não só os casais e as suas famílias, mas também a sociedade em geral.

\section{Referências}

Alarcão, M. (2006). (Des)equilibrios familiares. Coimbra: Quarteto Editora.

Blanchard, V. L., Hawkins, A. J., Baldwin, S. A., \& Fawcett, E. B. (2009). Investigating the effects of marriage and relationship education on couples' communication skills: A meta-analytic study.Journal of Family Psychology, 23, 203$-214$.

Bradbury, T. N., \& Fincham, F. D. (1990).Preventing marital dysfunction: Review and analysis. In F. D. Fincham \& T. N. Bradbury (Eds), The psychology of marriage: Basic issues and applications (pp. 375-399). New York: Guilford Press.

Brennan, J. (2001).Adjustment to cancer - coping or personal transition?Psycho-Oncology, 10, 1-18.

Bowling, T. K., Hill, C. M., \& Jencius, M. (2005).An overview of marriage enrichment.The Family Journal, 13, 87-94.

Bodenmann, G. (1997). Dyadic coping - a systemic-transactional view of stress and coping among couples: Theory and empirical findings. European Review of Applied Psychology, 47, 37-140.

Bodenmann, G. (2005). Dyadic coping and its significance for marital functioning. In T. A. Revenson, K. Kayser, \& G. Bodenmann (Eds), Couples coping with stress: Emerging perspectives on dyadic coping (pp. 33-49). Washington, DC: American Psychological Association.

Bodenmann, G. (2007). Dyadic coping inventory (DCI).Testmanual. Bern, Göttigen: Huber \& Hogrefe Tests.

Bodenmann, G., \& Shantinath, S. (2004). The couples coping enhancement training (CCET): A new approach to prevention of marital distress based upon stress and coping. Family Relations, 55, 477-484.

Christensen, A., \& Heavey, C. L. (1999). Interventions for couples.Annual Review Psychology, 50, 165-190.

Clarke, S. C. (1995). Advance report of final divorce statistics, 1989 and 1990. Monthly Vital Statistics Report, 43,1-30. Retirado a 25 de Abril, 2009, de http://www.cdc.gov/nchs/data/mvsr/supp/mv43_09s.pdf

Clements, M. L., Stanley, S. M, \& Markman, H. J. (2004). Before they said "I Do": Discriminating among marital outcomes over 13 years. Journal of Marriage and Family, 66, 613-626.

Conger, R. D., \& Conger, K. J. (2002). Resilience in Midwestern families: Selected findings from the first decade of a prospective, longitudinal study. Journal of Marriage and Family, 64, 361-373.

Duncan, S. F., Holman, T. B., \& Yang, C. (2007). Factors associated with involvement in marriage preparation programs. Family Relations, 56, 270-278. 
Fincham, F.D., Stanley, S. M., \& Beach, S. R. (2007). Transformative processes in marriage: An analysis of emerging trends. Journal of Marriage and Family, 69, 275-292.

Fitzpatrick, M. A. (1988).Between husbands and wives: Communication in marriage. Newbury Park, CA: Sage.

Floyd, F., Markman, H., Kelly, S., Blumberg, S., \& Stanley, S. (1995). Prevention: Conceptual, research, and clinical issues. In N. Jacobson \& A. Gurman (2nd ed.), Clinical handbook of marital therapy (pp. 173-195).New York: Guilford Press.

Gottman, J. M. (1993). A theory of marital dissolution and stability.Journal of Family Psychology, 7, 57-75.

Gottman, J. M. (1999). The marriage clinic: A scientifically based marital therapy. New York: W. W. Norton \& Company.

Gottman, J., \& Gottman, J (1999). The marriage survival kit. In R. Berger \& M.T. Hannah (Eds.), Preventive approaches in couples therapy (pp. 304-330). Philadelphia, PA: Brunner/Mazel.

Gottman, J. M., \& Gottman, J. S. (2006a).The art \& science of love: A workshop for couples. Seattle: The Gottman Institute.

Gottman, J. M., \& Gottman, J. S. (2006b). The brief Sound Relationship Questionnaires. In J. M. Gottman \& J. S, Gottman (eds.), The Art \& Science of love: A workshop for couples (pp. 291-300). Seattle: The Gottman Institute.

Gottman, J. M., \& Gottman, J. S. (2009). Gottman method couple therapy. In A. Gurman (4rd ed.), Clinical handbook of couple therapy (pp. 138-164). New York: Guilford Press.

Gottman, J. M., Gottman, J. S., \& DeClaire, J. (2006).Ten lessons to transform your marriage. New York: Crown Publishers.

Gottman, J. M. \& Silver, N. (2001). Os sete principios do casamento. Cascais: Editora Pergaminho.

Guerney, B. G. (1987). Relationship enhancement manual. Bethesda, MD: Ideal.

Gurman, A. (2009). Clinical handbook of couple therapy (4th ed.) New York: Guilford Press.

Gurman, A. S., \& Fraenkel, P. (2002). The history of couple therapy: A millennial review. Family Process, 41, 200-260.

Halford, W. K. (2004). The future of couple relationship education: Suggestions on how it can make a difference. Family Relations, 53, 559-566.

Halford, W. K., Kelly, A., \& Markman, H. J. (1997).The nature of healthy marriage.In W. K. Halford \& H. J. Markman (Eds.), Clinical handbook of marriage and couples intervention (pp. 145-160).Chichester: John Wiley \& Sons.

Halford, W. K., Markman, H. J., Kline, G. H., \& Stanley, S. M. (2003). Best practice in couple relationship education. Journal of Marital and Family Therapy, 29, 385-306.

Halford, W. K., Markman, H. J., \& Stanley, S. (2008). Strengthening couples' relationships with education: Social policy and public health perspectives.Journal of Family Psychology, 22, 497-505. 
Halford, W. K., \& Moore, E. N. (2002).Relationship education and the prevention of couple relationship problems.In A.S. Gurman \& N.S. Jacobson (3rd ed.), Clinical handbook of couple therapy (pp. 400-419). New York: Guilford Press.

Halford, W. K., Moore, E. M., Wilson, K. L., Dyer, C., \& Farrugia, C. (2004). Benefits of a flexible delivery relationship education: An evaluation of the couple CARE program. Family Relations, 53, 469-476.

Halford, W. K., \& Simons, M. (2005).Couple relationship education in Australia.Family Process, 44, 147-159.

Halford, W.K., Scott, J., \& Smythe, J. (2000).Couples and cancer.In K. Schmaling \& T. Sher (Eds.), Couples and illness (pp. 135-170). Washington DC: American Psychological Association.

Hahlweg, K., Markman, H. J., Thurmaier, F., Engl, J., \& Eckert, V. (1998). Prevention of marital distress: Results of a German prospective longitudinal study. Journal of Family Psychology, 12, 543-556.

Hawkins, A. J., Blanchard, V. L., Baldwin, A. S., \& Fawcett, E. B. (2008). Does marriage and relationship education work? A meta-analytic study.Journal of Consulting and Clinical Psychology, 76, 723-734.

Hendrick, C. (2002). A new age of prevention?Journal of Social and Personal Relationships, 19, 621-627.

Hendrick, S. S., Dicke, A., \& Hendrick, C. (1998). The relationship assessment scale.Journal of Social and Personal Relationship, 15, 137-142.

Holman, T. B., Larson, J. H., \& Harmer, S. L. (1994). The development and predictive validity of a new premarital assessment instrument: The preparation for marriage questionnaire, Family Relations, 43, 46-52.

Hunt, R. A., Hof, L., \& DeMaria, R. (1998). Marriage enrichment: Preparation, mentoring, and outreach. Philadelphia: Brunner/Mazel.

Jacobson, N. S., Schmaling, K. B. \& Holtzworth-Munroe, A. (1987). Component analysis of behavioral marital therapy: Two-year follow-up and prediction of relapse. Journal of Marital and Family Therapy, 13, 187-195.

Jakubowski, S.F., Milne, E. P., Brunner, H., Miller, R. B. (2004). A review of empirically supported marital enrichment programs.Family Relations, 53, 528$-536$.

Karney, B. J., \& Bradbury, T. N. (1995). The longitudinal course of marital quality and stability: A review of theory, method, and research. Psychological Bulletin, 118, 3-34.

Kayser, K., \& Scott, J. L. (2008). Why work with couples? In K. Kayser \& J.L. Scott (Eds.), Helping couples cope with women's cancers: An evidence-based approach for practitioners (pp. 35-51). New York: Springer

Larson, J. H., Holman, T. B., Klein, D. M., Busby, D. M, Stahmann, R. F., \& Peterson, D. (1995). A review of comprehensive questionnaires used in premarital education and counseling. Family Relations, 44, 245-252.

Lind, W. (2008). Casais biculturais e monoculturais: Diferenças e recursos. Tese de Doutoramento. Faculdade de Psicologia e de Ciências da Educação da Universidade de Lisboa. Retirado a 20 de Março, 2009, de http://hdl.handle.net/ $10451 / 977$ 
Lindahl, K. M., Malik, N. M., \& Bradbury, T. N. (1997). The development course of couples` relationships.In W. K. Halford, \& H. J. Markman (Eds.), Clinical handbook of marriage and couples intervention (pp. 203-223).Chichester: John Wiley \& Sons.

Markey, B., \& Micheletto, M. (1997).Instructor manual for FOCCUS. Omaha, NE: Archdiocese of Omaha.

Markman, H.J., Floyd, F. J., Stanley, S. M., \& Storaasli, R. D. (1988). Prevention of marital distress: A longitudinal investigation. Journal of Consulting and Clinical Psychology, 56, 210-217.

Markman, H. J., Halford, W. K., \& Cordova, A. D. (1997). A grand tour of future directions in the study and promotion of healthy relationships.In W. K. Halford \& H. J. Markman (Eds.), Clinical handbook of marriage and couples interventions (pp. 695-714). Chichester: John Wiley \& Sons.

Markman, H. J., \& Kraft, S. A. (1989). Men and women in marriage: Dealing with gender differences in marital therapy. The Behavior Therapist, 12, 51-56.

Miller, S., Miller, P., Nunnally, E. W., \& Wackman, D. B. (1992).Couple communicationinstructor manual. Evergreen, CO: International Communication Programs.

Narciso, I. (2001). Conjugalidades satisfeitas mas não perfeitas: À procura do padrão que liga. Tese de doutoramento não publicada. Faculdade de Psicologia e Ciências da Educação da Universidade de Lisboa.

Narciso, I. (2002). Janela com vista para intimidade. Psychologica, 31, 49-62.

Narciso, I., \& Ribeiro, M. T. (2009). Olhares sobre a conjugalidade. Lisboa: Coisas de Ler

Olsen, D. H., Fournier, D. H., \& Druckman, J. M. (1996). PREPARE. Minneapolis, MN: Life Innovations.

Perel, E. (2007). Mating in captivity: sex, lies and domestic bliss. United Kingdom: Hodder \& Stoughton.

Pihet, S., Bodenmann, G., Cina, A., Widmer, K., \& Shantinath, S. (2007). Can prevention of marital distress improve well-being? A 1 year longitudinal study.Clinical Psychology \& Psychotherapy, 14, 79-88.

Ribeiro, M. T. (2002). Da diversidade do masculino e do feminino à singularidade do casal. Tese de doutoramento não publicada. Faculdade de Psicologia e Ciências da Educação da Universidade de Lisboa.

Rogge, R. D., Cobb, R. M., Johnson, M., Lawrence, E., \& Bradbury, T. N. (2002). The CARE program: A preventive approach to marital intervention. In A. S. Gurman \& N. S. Jacobson (3rd ed.), Clinical handbook of couple therapy (pp. 420-435). New York: Guilford Press.

Ross, C. E. (1995). Reconceptualizing marital status as a continuum of social attachment. Journal of Marriage and the Family, 57, 129-140.

Ryan, K. D. \& Gottman, J. M. (s. d.). Psycho-educational intervention with moderately and severely distressed married couples - 1-year follow up results. Retirado a 15 Março, 2009, de http://www.gottman.com/files/Submit_Ryan Gottman\%20paper\%20_unhappy\%20vs.\%20severely_\%205-1-06.pdf. 
Silliman, B., Stanley, S. M., Coffin, W., Markman, H. J., \& Jordan, P. L. (2001). Preventive interventions for couples. In H. A. Liddle, D. A. Santisteban, R. F. Levant, \& J. H. Bray (Eds), Family psychology: Scientific based interventions (pp. 69-87). Washington D.C.: American Psychological Association.

Spitzberg, B. H. (2003). Methods of interpersonal skill assessment.In J. O. Greene \& B. R. Burleson (Eds.), Handbook of communication and social interaction skills (pp. 93-134). Mahwah, NJ: Erlbaum.

Stanley, S. M. (2001). Making a case for premarital education. Family Relations, 50, 272-280.

van Widenfelt, B., Markman, H. J., Guerney, B., Behrens, B. C., \& Hosman, C. (1997). Prevention of relationship problems.In W. K. Halford \& H.J. Markman (Eds.), Clinical handbook of marriage and couples (pp. 651-675). Chichester: John Wiley \& Sons.

Vedes, A. M., Lind, W. R., \& Lourenço, M. (2009). Factores de risco e resiliência na promoção da satisfação conjugal: Um caminho para a prevenção? Projecto de investigação de doutoramento não publicado. Faculdade de Psicologia da Universidade de Lisboa e Faculdade de Psicologia e Ciências da Educação da Universidade de Coimbra.

Veroff, J., Kulka, R. A., \& Douvan, E.(1981).Mental health in America. New York: Basic Books.

Vincent, J. P., Weiss, R. L., \& Birchler, G. R. (1975).A behavioral analysis of problem solving in distressed and nondistressed married and stranger dyads.BehaviorTherapy, 6, 475-487.

Watzlawick, P., Weakland, J., \& Fish, R. (1975).Change: principles of problem formation and problem resolution. New York: W. W. Norton \& Company.

World Health Organization (1998). Health promotion glossary. Geneva: Author. 